\title{
Geometry Education, Including the Use of New Technologies: A Survey of Recent Research
}

\author{
Nathalie Sinclair, Maria G. Bartolini Bussi, Michael de Villiers, \\ Keith Jones $\mathbb{D}$, Ulrich Kortenkamp, Allen Leung and Kay Owens
}

\begin{abstract}
This is a summary report of the ICME-13 survey on the theme of recent research in geometry education. Based on an analysis of the research literature published since 2008, the survey focuses on seven major research threads. These are the use of theories in geometry education research, the nature of visuospatial reasoning, the role of diagrams and gestures, the role of digital technologies, the teaching and learning of definitions, the teaching and learning of the proving process, and moves beyond traditional Euclidean approaches. Within each theme, there is commentary on promising future directions for research.
\end{abstract}

\footnotetext{
N. Sinclair

Simon Fraser University, Burnaby, Canada

e-mail: nathsinc@sfu.ca

M.G. Bartolini Bussi

Università di Modena e Reggio Emilia, Reggio Emilia, Italy

e-mail: mariagiuseppina.bartolini@unimore.it

M. de Villiers

University of Stellenbosch, Stellenbosch, South Africa

e-mail: profmd1@mweb.co.za

K. Jones $(\square)$

University of Southampton, Southampton, UK

e-mail: d.k.jones@ soton.ac.uk

U. Kortenkamp

University of Potsdam, Potsdam, Germany

e-mail: ulrich.kortenkamp@uni-potsdam.de
}

\author{
A. Leung \\ Hong Kong Baptist University, Hong Kong, Hong Kong SAR \\ e-mail: aylleung@hkbu.edu.hk \\ K. Owens \\ Charles Sturt University, Bathurst, Australia \\ e-mail: kowens@csu.edu.au \\ (C) The Author(s) 2017 \\ G. Kaiser (ed.), Proceedings of the 13th International Congress on Mathematical \\ Education, ICME-13 Monographs, DOI 10.1007/978-3-319-62597-3_18
}




\section{Introduction}

Recent research in geometry education was identified by the IPC of ICME-13 as being especially important and warranting an in-depth review pinpointing new knowledge, new perspectives, significant realisations and emerging challenges in a comprehensive and synthetic way, paying specific attention to the evolution since the previous ICME in 2012. In conducting the review (for a fuller report, see Sinclair et al., 2016), our first task was to identify major threads in the recent research literature relating to geometry education. We undertook this phase of work by generating a list of key threads in recent research based on our collective knowledge and experience. Each team member proposed a list of possible threads for consideration. Some suggestions were combined to produce a broader thread and for some there was not sufficient research literature on which to draw. This iterative process led to the identification of seven major research threads: the use of theories in geometry education research, the nature of visuospatial reasoning, the role of diagrams and gestures, the role of digital technologies, the teaching and learning of definitions, the teaching and learning of the proving process, and moves beyond traditional Euclidean approaches.

With the major research threads identified, pertinent research was identified in peer-reviewed journal articles, international peer-reviewed conference proceedings (such as PME and CERME) and leading research handbooks. For each thread, we produced a comprehensive annotated bibliography and this led to a further refinement in the detail of the research threads. While paying specific attention to research undertaken since 2012, we refer back to seminal publications including ones prior to 2008. In what follows, we summarize the key findings for each thread of the review survey, beginning with the use of theories in geometry education research. We conclude with a final section in which we highlight some overall issues and opportunities and discuss future directions for research.

\section{The Use of Theories in Geometry Education Research}

The development and refinement of theories of teaching and learning is one of the key aims of research in education in general, and for research in mathematics education in particular. In geometry education research, this focus on theory includes the developing and refining of theories that are specifically about the teaching and learning of geometry, as well as the application and development of more general theories to the specifics of geometry education.

Theories that are specifically about geometry education and that continue to be evident in research include the van Hiele model (1986), the theory of figural concepts (Fischbein, 1993; Mariotti \& Fischbein, 1997), and the theory of figural apprehension (Duval, 1998). Researchers are continuing to develop, refine and apply these theories. More recently, the theory of geometric work has been 
developed (c.f., Kuzniak, 2014). This aims at networking the theoretical ideas of figural, instrumental, and discursive geneses of geometric understanding by characterizing different geometrical paradigms and accounting for interaction between the epistemological and cognitive levels.

Theories being applied to geometry education include prototype phenomenon (Gal \& Linchevski, 2010), semiotic mediation (Bartolini Bussi \& Baccaglini-Frank, 2015; Bartolini Bussi \& Mariotti, 2008), variation (Gu, Huang, \& Marton 2004; Huang \& Li, 2016), the $\mathrm{cK} \varnothing$ (conception, knowing, concept) model (Balacheff, 2013), discursive, embodied, ecocultural and material perspectives ( $\mathrm{Ng} \&$ Sinclair, 2015a, b; Owens, 2014, 2015) and instrumental genesis (evident in research on the use of digital technologies) (Hegedus \& Moreno-Armella, 2010). These theoretical approaches illustrate the wide scope of geometry education research.

Overall, during the past decade, there has been increased focus on embodied and discursive theories in research on the teaching and learning of geometry, with a concomitant research emphasis on theories relating to visuospatial reasoning, the role of gestures and diagrams, and the use of digital technologies.

\section{The Nature of Visuospatial Reasoning}

Our review survey identified how attention is increasingly focusing on forms of visuospatial reasoning (Healy \& Powell, 2013; Owens, 2015; Rivera, 2011), variously referred to as visualization, visualising, spatial thinking, spatial reasoning, visuospatial thinking and visual reasoning. Here we use the term visuospatial reasoning to emphasize the spatial, visualizing (imagistic and as representations that others can see), and reasoning aspects of the visuospatial. While visuospatial reasoning is arguably relevant in all areas of mathematics, it has particular significance in the teaching and learning of geometry.

Overall, we can summarize our review survey on this thread by highlighting three developments. First, visualising (mentally and physically) is well-recognized as important in mathematics education but may not always be given sufficient emphasis in curriculum and teaching, perhaps because it is not straightforward to assess. Second, reasoning involves thinking about, and making decisions based on, visuospatial perception and understanding, both of which are influenced by prior knowledge and context of learning. Third, there is evidence that visuospatial reasoning in geometry can be improved through experience from perception to higher levels of reasoning (relevant here is the notion of cognitive malleability).

There are a number of educational implications of the recent research. First, the idea of locating: this is where younger children use geometric features and landmarks to find their way around larger spaces through spatial as well as visual perception for decision-making. Here, cultural studies support cognitive science studies. Second, the idea of transformation: here experience of mental rotation has been shown to improve algebraic manipulation, illustrating the value of spatial, as compared to object, visualizing. Overall, the value of a spatially-enriched education 
is being recognized more generally, including its value in counteracting the impact of gender, culture, experience, and capability differences.

Research increasingly emphasizes the need for good visuospatial working memory in geometry and more widely (Giofrè, Mammarella, Ronconi, \& Cornoldi, 2013). Classroom activities such as origami, pop-up engineering, quality block play, various practical activities, and specific forms of technology have all been shown to enhance visuospatial reasoning. Across the research domains of education and the cognitive sciences, there is converging agreement on the importance and malleability of visuospatial reasoning.

On top of this, while drawing provided evidence of learning (as well as being a mediating tool in learning), there is undoubted complexity of reasoning about visuals and different impacts of different representations. What is more, different activities create different imaginal, formational and transformational visuospatial reasoning. Alongside research on how the processes of visual perception and perception-based knowledge influence learning is evidence that the impact of Western-style education may limit visuospatial reasoning of indigenous, colonised groups while the use of technologies such as dynamic geometry environments (DGEs) can assist learning in developing communities as well as developed (Owens, 2015).

\section{The Role of Diagrams and Gestures in Geometry Education}

Research highlighting the role of diagrams and gestures has largely emerged out of recent emphases on the semiotic and embodied nature of geometry thinking and learning (e.g. Bartolini Bussi \& Baccaglini-Frank, 2015; Bartolini Bussi \& Mariotti, 2008; Ng \& Sinclair, 2015a, b). Here we consider historical-cultural perspectives that underscore the role of semiotic processes and artefacts in geometry teaching and learning. In doing so, we highlight embodiment perspectives that stress the important roles of gestures and diagrams in geometry teaching and learning.

In addition to research with school-aged pupils, researchers have begun to study the role of gestures and diagrams in the work of professional mathematicians (Barany \& MacKenzie, 2014; Hare \& Sinclair, 2015). This work corroborates some of the claims of Châtelet (2000) that diagrams are more than representations of existing knowledge while also providing more detailed and real-time evidence of the meanings that gesturing and diagramming help to create, even in highly advanced mathematics.

This work, when combined with the studies noted above on the semiotic and embodied nature of geometry thinking and learning, provides a clear indication of the importance of encouraging learners to engage in more gesturing and diagramming. Existing research (referenced above) suggests that the more teachers gesture, the more do their students. Future research could provide insight into the types of gestures that might be helpful, as well as the modalities in which students 
are invited to gesture. Gestures, imitation, and explanation are also important in indigenous communities making use of their strong capabilities in visuospatial reasoning (c.f., Owens, 2015).

\section{The Role of Digital Technologies in Geometry Education}

New technological developments over the past decade have led to new challenges in the use of technology in the teaching and learning of geometry. This is despite the role of technologies in teaching and learning not being understood completely, nor being explored in sufficient detail since the introduction of DGEs (such as Cabri-Géomètre and Geometer's Sketchpad) in the early 1990s. As such, this demonstrates the importance of three areas of research: (1) the introduction and design of new technology, both hardware and software, (2) theory and methodology for a better understanding of the role of existing and emerging technology, and (3) empirical studies on the use of technology in teaching and learning.

Technology in geometry education has become relatively mainstream, yet there is still not enough research into its specific effects. This is due, in part, to the way that some technologies, such as DGEs, change geometric representations and discourse quite significantly, as compared with paper-and-pencil approaches. As a result, articulation in the classroom, with textbooks, physical manipulatives, and especially assessment (Venturini \& Sinclair, 2016) can be quite challenging.

Trends for digital geometry tools include (1) how geometry on the web is encountering various technological difficulties associated with the need to replace Java; (2) the issue of interface design and how users interact with onscreen geometry (Jackiw \& Sinclair, 2009; Kortenkamp \& Dohrmann, 2010; Laborde \& Laborde, 2014; Mackrell 2011; Schimpf \& Spannagel, 2011; (3) the rise of mobile devices and touch technology; and (4) new modes of interaction such as multi-touch and multi-collaboration.

In terms of specific digital tools and concepts, there are many issues-ranging from detailed matters such as the use of onscreen 'sliders' in geometry to wider socio-cultural aspects such as how technology has social impact. Across geometry education, the issues range from the role of digital technologies in developing learners' spatial capabilities and capability with 3D geometry (the latter being available digitally but currently restricted to $2 \mathrm{D}$ projection) to the issue of task design and how tasks change with availability of technology (Leung \& Baccaglini-Frank, 2016). On top of this, there are issues of assessment, feedback and learning analytics, where new approaches are emerging. What is more, teacher education and professional development continue as challenging and important tasks for the mathematics education community in general, and for geometry educators in particular.

The role of technology is just beginning to be understood. At the same time, technology continues to evolve and rapidly change the everyday world and the 
classroom. Students and teachers are increasingly using digital tools throughout the day (and beyond school, Carreira, Jones, Amado, Jacinto, \& Nobre, 2016). It is increasingly necessary to understand better how new and emerging digital tools can be used effectively.

\section{The Teaching and Learning of Geometric Definitions}

The importance of definitions in geometry education (c.f. de Villiers, Govender, \& Patterson, 2009; Mariotti \& Fischbein, 1997; Smith, 2010) is reflected in the research literature, with a number of studies on this theme appearing over the past decade. Such research has focused both on understanding the process of defining and on the need for definitions. Overall, the majority of studies have concentrated on descriptive (a posteriori) defining; for example, defining circles, triangles, quadrilaterals, and polyhedra after exploring their properties with the use of DGEs, paper-folding, and/or pencil-and-paper construction (c.f. Choi and Oh 2008; Fujita \& Jones, 2007; Salinas, Lynch-Davis, Mawhinney, \& Crocker, 2014; Usiskin, Griffin, Witonsky, \& Willmore, 2008; Zandieh \& Rasmussen, 2010).

It appears that the fundamental issue of understanding the need for axioms and for accepting some statements as definitions to avoid circularity has been largely under-researched in the mathematics education community (though see Fujita, Jones, \& Miyazaki, 2011; Miyazaki, Fujita, \& Jones, 2017). Another under-researched area seems to be exploring the existence of a mathematical choice between defining (and classifying) the quadrilaterals hierarchically or in partitions (compare de Villiers 1994; Usiskin et al. 2008). A specific research question in this regard might be the extent to which students and teachers understand (or how to develop such understanding) that choosing a hierarchical definition over a partition leads to a more economical (shorter) definitions more concise formulation of some theorems, simplified deductive structure (by decreasing the number of proofs required), assists in problem solving, etc.

The potential of DGEs, and some use of analogy also, in developing understanding for definitions have been explored in several studies with triangles and quadrilaterals (c.f. Kaur, 2015). Such approaches appear to have assisted students in developing more robust, dynamic concept images than the traditional prototypical, static images that tend to prevent inclusive definitions. Nevertheless, everyday language and prototypical conceptions remain an issue especially in regard to class-inclusion as well as students' understanding the constraints of a DGE figure. A paucity of research on the use of symmetry concepts in choice of definitions, and on engaging students in the process of the constructive (a priori) defining of new concepts, means that these are ripe areas for future research. 


\section{The Teaching and Learning of the Proving Process in Geometry}

Much research over the past decade has focused on studying the teaching and learning of the proving process, particularly in light of the increasing use of educational technology. Researchers have turned their attention to the following issues, many of them of perennial interest: what is and what constitutes a mathematical proof; how to interpret proof as an explanation that convinces others, and what makes something convincing; what kind of pedagogy and pedagogical tools are conducive to the construction of proof; and so on.

In terms of what is and what constitutes a mathematical proof, recent studies suggest that (geometrical) proof is bounded socio-culturally and is intimately related to the perceptual world. In terms of alternative frameworks for what is a 'geometrical proof', research suggests this is closely tied with the corresponding conjecture or hypothesis; in particular, with how the conjecture or hypothesis came about. In that, empirical-based argument may play a role in the formation of geometrical proof with respect to convincing or explaining.

The use of DGEs has been playing a vital epistemic role in studies that have probed the process of generating geometrical conjectures (c.f. Leung, 2008; Leung, Baccaglini-Frank, \& Mariotti, 2013). For example, through the lens of the theory of semiotic mediation (TSM), the conjecture production process is a semiotic process that involved a transformation from personal signs to mathematical signs. Here, feedback and mediation from the technological tool serve as means for boundary crossing between the empirical and theoretical contexts in the proving process. In particular, the DGE drag-mode instigates the complex interplays between inductive, abductive and deductive reasoning in the transition between empirical and theoretical proof perspectives. Studying and categorizing DGE dragging modalities/strategies have been a core focus attempting to conceptualize proof and explanation when using a DGE. Studies have explored the role of DGE as an epistemic tool, in particular dragging, to open up a quasi-empirical dimension to the nature of proof, even indirect proof.

Pedagogies such as tool-based task design, inquiry-based learning, mathematical discussion, problem modification, geometrical construction, and a focus on gesturing, have been introduced to improve the conjecture formation processes. This has included the following: use of the shift-problem approach (empirical proof schemes, external conviction proof schemes, and deductive proof schemes); modification of textbook problems into DGE investigations; use of flow-chart proving (Miyazaki, Fujita, \& Jones, 2015); use of the lens of cognitive unity to address the tension between carrying out a geometrical construction and constructing the related proof; and the interplay among gestures, discourse and diagram in geometric reasoning. 


\section{Moves Beyond Traditional Euclidean Approaches to Geometry}

In the teaching and learning of 3D geometry, research indicates that students exhibit similar prototypical predispositions to 3D objects as they do with $2 \mathrm{D}$ objects (c.f. Sarfaty \& Patkin, 2013). Physically building, constructing and drawing 3D objects such as polyhedra, and/or exploring them dynamically with 3D DGEs appear to develop better concept images and understanding of their properties. There are few studies on engaging students in extending interesting 2D results to $3 \mathrm{D}$; for example, triangle concurrencies, Pythagoras, Varignon's or Viviani's theorem. The use of analogy when moving from $2 \mathrm{D}$ to $3 \mathrm{D}$ and higher dimensions could be more extensively explored using analogous concepts for triangle, square, circles, perpendicular bisector, angle bisector, etc.

Experimental studies on spherical and hyperbolical surfaces have used specific manipulatives such as spheres or DGEs to explore and prove results and properties of non-Euclidean objects, and in most cases, contrasting/comparing them with equivalents from Euclidean geometry. Guven and Baki (2010) theorised van Hiele levels of understanding for spherical geometry similar to $2 \mathrm{D}$, which appeared to be reasonably confirmed by a Guttman scalogram analysis, though future studies would be useful. There have been studies focused on a Turtle geometry model of the hyperbolic surface (Arzarello, Bartolini Bussi, Leung, Mariotti, \& Stevenson, 2012) and on topological surfaces (the Mobius strip, the torus and the Klein bottle) using a DGE (Hawkins \& Sinclair, 2008). In contrast, there has been little or no research on the teaching and learning of fractals over the past 10 years.

\section{Concluding Comments and Future Directions}

The seven themes that we identified in our survey review (aided by survey team member Alexey Zaslavsky, Russia, and ICME-13 IPC liaison Behiye Ubuz, Turkey) reflect both traditional research interests in the teaching and learning of geometry as well as new areas of growth. During the past decade, there has been increased focus on embodied and discursive theories in research on the teaching and learning of geometry, with a concomitant research emphasis on visuospatial reasoning, on the use of gestures and diagrams and on digital tools. The effectiveness of certain digital tools, such as DGES, as well as their increased availability, has also affected researched on topics that span the k-16 geometry curriculum (from early experiences with dynamic triangles to later explorations in spherical geometry) as well as major areas of research such as the proving process and the use and role of definitions. There has also been a broadening of the traditional scope of geometry, both in terms of cultural perspectives and also in terms of concepts and activities that do not follow the typical Euclidean development-including the Euclidean approach to definitions. 
We expect to see continued growth in these areas, and we also hope to see increased research interest in the teaching and learning of geometry since it is a topic whose significance may have become under-recognized through an increased policy emphasis on number and algebra. A valuable focus of future research might be to investigate how geometric ways of thinking, including visuospatial reasoning and diagramming, may serve not only to improve geometric understanding, but also mathematical understanding more generally, and may even broaden the range of learners who might become interested in, and excel at, mathematics.

\section{References}

Arzarello, F., Bartolini Bussi, M. G., Leung, A., Mariotti, M. A., \& Stevenson, I. (2012). Experimental approach to theoretical thinking. In G. Hanna \& M. de Villers (Eds.), Proof and proving in mathematics education (pp. 97-137). New York: Springer.

Balacheff, N. (2013). cKф, a model to reason on learners' conceptions. In M. V. Martinez \& A. C. Superfine (Eds.), Proceedings of PME-NA 35 (pp. 2-15). IL, United States: Chicago.

Barany, M., \& MacKenzie, D. (2014). Chalk: Materials and concepts in mathematics research. In C. Coopmans, J. Vertesi, M. Lynch, \& S. Woolgar (Eds.), Representation in scientific practice revisited (pp. 107-130). Cambridge, MA: MIT Press.

Bartolini Bussi, M. G., \& Baccaglini-Frank, A. (2015). Geometry in early years: Sowing the seeds towards a mathematical definition of squares and rectangles. ZDM: International Journal on Mathematics Education, 47(3), 391-405.

Bartolini Bussi, M. G., \& Mariotti, M. A. (2008). Semiotic mediation in the mathematics classroom: Artefacts and signs after a Vygotskian perspective. In L. English et al. (Eds.), Handbook of international research in mathematics education (pp. 720-749). Mahwah, NJ: Erlbaum.

Carreira, S., Jones, K., Amado, N., Jacinto, H., \& Nobre, S. (2016). Youngsters solving mathematical problems with technology. New York: Springer.

Châtelet, G. (2000). Figuring space: Philosophy, mathematics, and physics. Dordrecht: Kluwer.

Choi, K., \& Oh, S. K. (2008). Teachers' conceptual errors related to the definitions in the area of geometry of elementary school mathematics. Journal of the Korean Society of Mathematical Education. Series A. The Mathematical Education, 47(2), 197-219.

de Villiers, M. (1994). The role and function of a hierarchical classification of quadrilaterals. For the Learning of Mathematics, 14(1), 11-18.

de Villiers, M., Govender, R., \& Patterson, N. (2009). Defining in geometry. In T. Craine \& R. Rubinstein (Eds.), Understanding geometry for a changing world (pp. 189-203). Reston: NCTM.

Duval, R. (1998). Geometry from a cognitive point of view. In C. Mammana \& V. Villani (Eds.), Perspectives on the teaching of geometry for the 21st Century: An ICMI study (pp. 37-52). Dordrecht: Kluwer.

Fischbein, E. (1993). The theory of figural concepts. Educational studies in mathematics, 24(2), $139-162$.

Fujita, T., \& Jones, K. (2007). Learners' understanding of the definitions and hierarchical classification of quadrilaterals: Towards a theoretical framing. Research in Mathematics Education, 9, 3-20.

Fujita, T., Jones, K., \& Miyazaki, M. (2011). Supporting students to overcome circular arguments in secondary school mathematics. Proceedings of PME35 (Vol. 2, pp. 353-60).

Gal, H., \& Linchevski, L. (2010). To see or not to see: Analyzing difficulties in geometry from the perspective of visual perception. Educational Studies in Mathematics, 74(2), 163-183. 
Giofrè, D., Mammarella, I. C., Ronconi, L., \& Cornoldi, C. (2013). Visuospatial working memory in intuitive geometry, and in academic achievement in geometry. Learning and Individual Differences, 23, 114-122.

Gu, L., Huang, R., \& Marton, F. (2004). Teaching with variation. In L. Fan et al. (Eds.), How Chinese learn mathematic (pp. 309-345). Singapore: World Scientific.

Guven, B., \& Baki, A. (2010). Characterizing student mathematics teachers' levels of understanding in spherical geometry. International Journal of Mathematical Education in Science and Technology, 41(8), 991-1013.

Hare, A., \& Sinclair, N. (2015). Pointing in an undergraduate abstract algebra lecture: Interface between speaking and writing. Proceedings of PME39 (vol. 3, pp. 33-40).

Hawkins, A., \& Sinclair, N. (2008). Explorations with Sketchpad in topogeometry. International Journal of Computers for Mathematical Learning, 13(1), 71-82.

Healy, L., \& Powell, A. (2013). Understanding and overcoming 'disadvantage' in learning mathematics. In M. Clements et al. (Eds.), Third international handbook of mathematics education (pp. 69-100). New York: Springer.

Hegedus, S. J., \& Moreno-Armella, L. (2010). Accommodating the instrumental genesis framework within dynamic technological environments. For the Learning of Mathematics, $30(1), 26-31$.

Huang, R., \& Li, Y. (Eds.). (2016). Teaching and learning mathematics through variation. Rotterdam: Sense.

Jackiw, N., \& Sinclair, N. (2009). Sounds and pictures: Dynamism and dualism in dynamic geometry. ZDM: International Journal on Mathematics Education, 41(4), 413-426.

Kaur, H. (2015). Two aspects of young children's thinking about different types of dynamic triangles. ZDM: International Journal on Mathematics Education, 47(3), 407-420.

Kortenkamp, U., \& Dohrmann, C. (2010). User interface design for dynamic geometry software. Acta Didactica Napocensia, 3(2), 59-66.

Kuzniak, A. (2014). Understanding the nature of the geometric work through its development and its transformation. In S. Rezat, M. Hattermann, \& A. Peter-Koop (Eds.), Transformation: A fundamental idea of mathematics education (pp. 311-325). New York: Springer.

Laborde, C., \& Laborde, J.-M. (2014). Dynamic and tangible representations in mathematics education. In S. Rezat, M. Hattermann, \& A. Peter-Koop (Eds.), Transformation: A fundamental idea of mathematics education (pp. 187-202). New York: Springer.

Leung, A. (2008). Dragging in a dynamic geometry environment through the lens of variation. International Journal of Computers for Mathematical Learning, 13(2), 135-157.

Leung, A., \& Baccaglini-Frank, A. (Eds.). (2016). Digital technologies in designing mathematics education tasks. New York: Springer.

Leung, A., Baccaglini-Frank, A., \& Mariotti, M. A. (2013). Discernment in dynamic geometry environments. Educational Studies in Mathematics, 84(3), 439-460.

Mackrell, K. (2011). Design decisions in interactive geometry software. ZDM: International Journal on Mathematics Education, 43(3), 373-387.

Mariotti, M. A., \& Fischbein, E. (1997). Defining in classroom activities. Educational Studies in Mathematics, 34, 219-248.

Miyazaki, M., Fujita, T., \& Jones, K. (2015). Flow-chart proofs with open problems as scaffolds for learning about geometrical proof. ZDM: International Journal on Mathematics Education, 47(7), 1211-1224.

Miyazaki, M., Fujita, T., \& Jones, K. (2017). Students' understanding of the structure of deductive proof. Educational Studies in Mathematics, 94(2), 223-239.

Ng, O., \& Sinclair, N. (2015a). Young children reasoning about symmetry in a dynamic geometry environment. ZDM: International Journal on Mathematics Education, 47(3), 421-434.

Ng, O., \& Sinclair, N. (2015b). 'Area without numbers': Using Touchscreen dynamic geometry to reason about shape. Canadian Journal of Science, Mathematics and Technology Education, 15(1), 84-101.

Owens, K. (2014). Diversifying our perspectives on mathematics about space and geometry. International Journal of Science and Mathematics Education, 12(4), 941-974. 
Owens, K. (2015). Visuospatial reasoning: An ecocultural perspective for space, geometry and measurement education. New York: Springer.

Rivera, F. (2011). Towards a visually-oriented school mathematics classrooms: Research, theory, practice, and issues. New York: Springer.

Schimpf, F., \& Spannagel, C. (2011). Reducing the graphical user interface of a dynamic geometry system. ZDM: International Journal on Mathematics Education, 43(3), 389-397.

Salinas, T. M., Lynch-Davis, K., Mawhinney, K. J., \& Crocker, D. A. (2014). Exploring quadrilaterals to reveal teachers' use of definitions: Results and implications. Australian Senior Mathematics Journal, 28(2), 50-59.

Sarfaty, Y., \& Patkin, D. (2013). The ability of second graders to identify solids in different positions and to justify their answer. Pythagoras, 34(1), 1-10.

Sinclair, N., Bartolini Bussi, M., de Villiers, M., Jones, K., Kortenkamp, U., Leung, A., et al. (2016). Recent research on geometry education: An ICME-13 Survey Team report. ZDM: International Journal on Mathematics Education, 48(5), 691-719.

Smith, J. T. (2010). Definitions and non-definability in geometry. American Mathematical Monthly, 117(6), 475-489.

Usiskin, Z., Griffin, J., Witonsky, D., \& Willmore, E. (2008). The classification of quadrilaterals: A study of definition. Charlotte, NC: InfoAge.

van Hiele, P. M. (1986). Structure and insight: A theory of mathematics education. New York: Academic Press.

Venturini, M., \& Sinclair, N. (2016). Designing assessment tasks in a dynamic geometry environment. In A. Leung \& A. Baccaglini-Frank (Eds.), Digital technologies in designing mathematics education tasks (pp. 77-98). New York: Springer.

Zandieh, M., \& Rasmussen, C. (2010). Defining as a mathematical activity. Journal of Mathematical Behavior, 29, 55-75.

Open Access Except where otherwise noted, this chapter is licensed under a Creative Commons Attribution 4.0 International License. To view a copy of this license, visit http://creativecommons. org/licenses/by/4.0/.

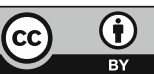

